\title{
Does reaction-diffusion support the duality of fragmentation effect?
}

\author{
Lionel Roques $^{\mathrm{a}, *}$, and M.D. Chekroun ${ }^{\mathrm{b}}$ \\ ${ }^{a} U R 546$ Biostatistique et Processus Spatiaux, INRA, F-84000 Avignon, France \\ ${ }^{\mathrm{b}}$ Environmental Research and Teaching Institute, École Normale Supérieure, \\ 24 rue Lhomond, 75231 Paris Cedex 05, France
}

\begin{abstract}
There is a gap between single-species model predictions, and empirical studies, regarding the effect of habitat fragmentation per se, i.e., a process involving the breaking apart of habitat without loss of habitat. Empirical works indicate that fragmentation can have positive as well as negative effects, whereas, traditionally, single-species models predict a negative effect of fragmentation. Within the class of reaction-diffusion models, studies almost unanimously predict such a detrimental effect. In this paper, considering a single-species reaction-diffusion model with a removal - or similarly harvesting - term, in two dimensions, we find both positive and negative effects of fragmentation of the reserves, i.e. the protected regions where no removal occurs. Fragmented reserves lead to higher population sizes for time-constant removal terms. On the other hand, when the removal term is proportional to the population density, higher population sizes are obtained on aggregated reserves, but maximum yields are attained on fragmented configurations, and for intermediate harvesting intensities.
\end{abstract}

Key words: fragmentation, single-species model, reaction-diffusion, harvesting, spatial patterns, conservation biology

\section{Introduction}

The analysis of the effects of environmental fragmentation and variability on population densities and biodiversity has stimulated the development of many

\footnotetext{
* Corresponding author. Fax: +33 432722182.

Email address: lionel.roques@avignon.inra.fr (Lionel Roques).
} 
spatially-explicit population models. In the modeling literature, positive effects of environmental variability have been recorded (see e.g. Bolker, 2003; Berestycki et al., 2005a). On the other hand Fahrig (2003), in a thorough bibliography analysis, pointed out that most single-species modeling approaches lead to comparable conclusions regarding the detrimental effects of fragmentation per se, i.e., a process involving the breaking apart of habitat without loss of habitat. She noted that unlike the effects of habitat loss (see Saunders et al., 1991, for a discussion of the consequences of fragmentation with habitat loss), and in contrast to current theory, empirical studies suggest that the effects of fragmentation per se are at least as likely positive as negative. The aim of this note is to make steps towards a reconciliation between the theory and empirical works on the effects of fragmentation, within the framework of reaction-diffusion models.

Reaction-diffusion models (hereafter RD models), although they sometimes bear on simplistic assumptions such as infinite velocity assumption and completely random motion of animals (Holmes, 1993), are not in disagreement with certain dispersal properties of populations observed in natural as well as experimental ecological systems, at least qualitatively (see Shigesada and Kawasaki, 1997; Turchin, 1998; Murray, 2002; Okubo and Levin, 2002). Furthermore, these models often provide a good framework for rigorous investigation of theoretical questions and derivation of qualitative as well as numerical results on population dynamics. In that respect, the effects of environmental fragmentation have been addressed in many theoretical studies based on such models over the last decades.

Within the class of RD models with heterogenous coefficients, numerous recent works have emphasized the detrimental effect of environmental fragmentation per se on species persistence and spreading, in agreement with the other theoretical tendencies noted by Fahrig. In all these RD models, the population growth rate function at a location $\mathbf{x}, r(\mathbf{x})$, was not constant, taking higher values in favorable regions than in unfavorable ones. Depending on the spatial arrangements of these regions, the modeled populations were shown to tend to extinction or survive, and to disperse at different speeds. In the particular case of 1-dimensional binary environments (i.e. for $r$ taking values in a set constituted of two values), Cantrell and Cosner (1989, 2003) and Shigesada and Kawasaki (1997) have established that concentrating all the habitat in a single patch improved persistence. Berestycki et al. (2005a) generalized these analytical results to the $N$-dimensional case, with more general growth rate functions. More recently Roques and Stoica (2007) carried out more precise results regarding the negative correlation between persistence and fragmentation. Habitat fragmentation has also been shown, first numerically (Kinezaki et al., 2003), and then analytically (El Smaili et al., 2009) to negatively affect population spreading speed. 
Nevertheless, to our knowledge, there do not exist detailed demonstration of positive effects related to fragmentation derived from RD models in two spacedimensions, although in a related work, Neubert (2003) shows positive effects of reserve fragmentation from RD models with harvesting terms, in the sense that fragmented reserves sometimes maximize the yield. However, in his work, which is carried out in a one-dimensional space, no explicit conservation of the area of the reserve is assumed.

In this paper, we study single-species RD models in two-space dimensions with a spatially-homogeneous growth term, and spatially heterogeneous removal terms $-Y$ which can be, for instance, interpreted as harvesting terms. The regions where $Y \equiv 0$ hence correspond to protected regions or similarly reserves. In our models, $Y=Y(\mathbf{x}, u)$ depends on the location $\mathbf{x}$, and can depend on the population density $u$ at this location. Typically, when $Y$ does not depend on the population density, it corresponds to a constant-yield harvesting strategy. In this case, a constant number of individuals are removed per unit of time. This is the case when a quota is set on the harvesters (Robinson and Redford, 1991; Robinson and Bodmer, 1999; Stephens et al., 2002). Even in the absence of such imposed quotas, harvesters often increase their effort to maintain a constant yield. A good example is provided by the high-trophic level fishes catches data in the North Atlantic reviewed by Christensen et al. (2003), which describe a decline of the biomass of one half from 1950 to 1990, while the catch remains the same. The function $Y$ can also be taken to be proportional to the density $u$, corresponding to a proportional harvesting strategy. Then, locally, a constant proportion of the population is removed per unit of time, corresponding to a constant effort of the harvesters. These two harvesting strategies have been investigated by the authors (Roques and Chekroun, 2007), in inhomogeneous environments. It was shown, through analytical and numerical analysis, that aggregated habitat configurations gave better chances of population persistence, respecting the tendency found in the modeling literature; see also Oruganti et al. (2002) for other mathematical results on these models.

We conduce here a different analysis. Not only we investigate the effects of the spatial arrangement of the harvesting term rather than those of the growth function, but we also focus on other quantities than simple persistence. Considering protected regions with a fixed total area but with gradually fragmented shapes, we analyze the intertwined effects of fragmentation and harvesting intensity on both the population size and the quantity of harvested individuals. To do that we use the stochastic model of landscape generation of Roques and Stoica (2007), that is in complete agreement with the concept of fragmentation per se, and we show that fragmentation of protected regions can in fact be beneficial to the modeled population and to the harvesters. These results demonstrate that two-dimensional $\mathrm{RD}$ models with harvesting terms can support a dual effect of fragmentation per se, positive as well as negative. 
Typically, it is shown, for instance, that fragmented reserves lead to higher population sizes for time-constant removal terms. On the other hand, when the removal term is proportional to the population density, higher population sizes are obtained on aggregated reserves, but maximum yields are attained on fragmented configurations, and for intermediate harvesting intensities.

\section{Materials and methods}

\subsection{The model}

The idea of modeling population dynamics with reaction-diffusion models has begun to develop at the beginning of the 20th century, with random walk theories of organisms, introduced by Pearson and Blakeman (1906). Then, Fisher (1937) and Kolmogorov et al. (1937) used a reaction-diffusion equation with homogeneous coefficients as a model for population genetics. Later, Skellam (1951) examined this type of model, and he succeeded to propose quantitative explanations of observations for the spread of muskrats throughout Europe at the beginning of 20th Century. Since then, these models have been widely used to explain spatial propagation or spreading of biological species (bacteria, epidemiological agents, insects, fishes, mammal, plants, etc., see the books Shigesada and Kawasaki, 1997; Turchin, 1998; Murray, 2002; Okubo and Levin, 2002, for review).

Ignoring age or stage structures as well as delay mechanisms or Allee effects, the classical Fisher-Kolmogorov model, in two space-dimensions, can be written as follows:

$$
\frac{\partial u}{\partial t}-D \nabla^{2} u=r u(1-u / K), t>0, \mathbf{x} \in \Omega \subset \mathbb{R}^{2},
$$

where $u=u(t, \mathbf{x})$ corresponds to the population density at time $t$ and position $\mathbf{x}=\left(x_{1}, x_{2}\right)$. The left-hand side of (11) corresponds to the diffusion equation, and simply describes the redistribution of organisms following uncorrelated random walks where $\nabla^{2}$ stands for the spatial dispersion operator $\nabla^{2} u=\frac{\partial^{2} u}{\partial x_{1}^{2}}+\frac{\partial^{2} u}{\partial x_{2}^{2}}$. The diffusion coefficient $D$ measures the individuals rate of movement, $r>0$ is the intrinsic growth rate of the population and $K>0$ corresponds to the environment carrying capacity.

The domain $\Omega$ is considered bounded, and we assume reflecting boundary conditions:

$$
\frac{\partial u}{\partial n}(t, \mathbf{x})=0, \text { for } \mathbf{x} \in \partial \Omega,
$$


where $\partial \Omega$ is the domain's boundary and $n=n(\mathbf{x})$ corresponds to the outward normal to this boundary. Thus, some part of the boundary can be considered as an absolute barrier that the individuals do not cross, like coasts, and other parts of the boundary can be seen as regions where as much individuals exit the domain as individuals enter the domain.

At this stage no environmental fragmentation is present in this class of models. To introduce it, we adopt a perturbative approach which consists in subtracting a spatially-dependent term to the right-hand side of equation (11):

$$
\frac{\partial u}{\partial t}-D \nabla^{2} u=r u(1-u / K)-Y(\mathbf{x}, u), t>0, \mathbf{x} \in \Omega \subset \mathbb{R}^{2}
$$

It could seem artificial to keep constant the parameters $r, D$ and $K$ whereas the removal term $Y$ is spatially-dependent. The ecological interpretation of such a framework is no more than the consideration of heterogeneously distributed harvesting in homogeneous media, a classical set-up in fisheries for instance. In fact, even in the case of a population living in a heterogeneous environment, the objective of such assumptions is to separate the effects in order to facilitate the ecological interpretations; and keeping constant the biological parameters can be thought as to consider the effects of spatially-dependent perturbations on an averaged model of growth (1), where the parameters are averaged in space and time. Advanced mathematical theory of averaging for partial differential equations can then be used to support this strategy and to make robust conclusions derived by such approach, at least in the case of small amplitude of oscillating parameters $r(t, \mathbf{x}), D(t, \mathbf{x})$ and $K(t, \mathbf{x})$ (see e.g. Hale and Verduyn Lunel, 1990; Chekroun and Roques, 2006) .

In the forthcoming computations, we assume that harvesting starts on a previously not harvested population, which has reached its stable positive steady state. In other words, the environment is assumed to have reached its carrying capacity at $t=0$, the time at which harvesting is started, with $u(0, \mathbf{x})=K$, where $u$ is the solution of (2). This assumption is a natural one for studying the destabilizing effects due to removal terms. Our model (2) being introduced and discussed, the analysis of environmental effects brought by the removal term $Y$ is addressed according two harvesting strategies that we present now.

\subsection{Harvesting strategies}

The first type corresponds to a "quasi"-constant-yield harvesting, with a removal term

$$
Y(\mathbf{x}, u)=\delta \cdot \chi(\mathbf{x}) \rho_{\varepsilon}(u)
$$


This model is close to but different from the so-called threshold harvesting model found in ordinary differential equations (ODEs) models of harvesting (see Roques and Chekroun, 2007).

In (3), $\chi(\mathbf{x})$ is a function taking the value 1 if $\mathbf{x}$ belongs to a harvested region, and the value 0 if $\mathbf{x}$ belongs to a protected region. It constitutes therefore what we call the harvesting field, with $\delta$ a positive constant which corresponds to the harvesting intensity in this field. The parameter $\delta$ can also be interpreted as a quota. The last term $\rho_{\varepsilon}(u)$ is a density-dependent threshold function:

$$
\rho_{\varepsilon}(s)=0 \text { if } s \leq 0, \rho_{\varepsilon}(s)=s / \varepsilon \text { if } 0<s<\varepsilon \text {, and } \rho_{\varepsilon}(s)=1 \text { if } s \geq \varepsilon,
$$

where $\varepsilon$ is a small threshold below which harvesting is progressively withdrawn. With such a harvesting function, at each location $\mathbf{x}$, the yield is constant in time whenever $u(t, \mathbf{x}) \geq \varepsilon$. Note that from a mathematical point of view, the function $\rho_{\varepsilon}$ ensures the nonnegativity of the solutions of (2) (Roques and Chekroun, 2007). Considering constant-yield harvesting functions without this threshold value would be unrealistic since it would lead to harvest on zero-populations.

The second type of strategy corresponds to a more standard proportional harvesting situation (Neubert, 2003), in a spatial context, where

$$
Y(\mathbf{x}, u)=E \cdot \chi(\mathbf{x}) u .
$$

The function $\chi$ is defined as above, and the term $E \cdot \chi(\mathbf{x})$ can now be interpreted as a harvesting effort at the location $\mathbf{x}$. The instantaneous yield at a point $\mathbf{x}$ is then proportional to this effort and to the local population density.

\subsection{The model of fragmentation}

There exist several ways of obtaining hypothetical landscape distributions, see e.g. Gardner et al. (1987) and Keitt (2000) for neutral landscape models, and Mandelbrot (1982) for measures of fragmentation based on fractal dimension. The model retained here is the one developed by Roques and Stoica (2007), and inspired from statistical physics. This is a neutral landscape model in the sense that it is a stochastic model of landscape pattern, and the value - protected or harvested in the present case - assigned to a position in the pattern is a random variable. As key property, this stochastic model provides a numerical procedure for generating several samples of landscapes with breaking apart of the habitat while keeping constant habitat abundance, along with an exact control of the later and the type of breaking apart. This model offers therefore an appropriate framework for assessing the effect of fragmentation per se on RD models, in the sense underlined by Fahrig (2003). We make precise here the main parameters calibrating the model for our present purpose. 


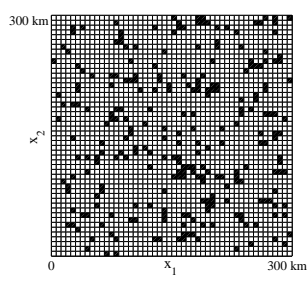

(a) $s(\chi)=94$

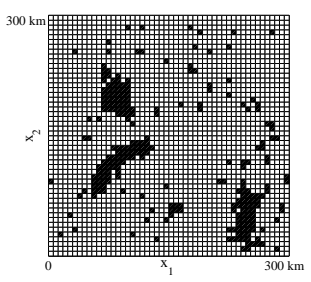

(b) $s(\chi)=274$

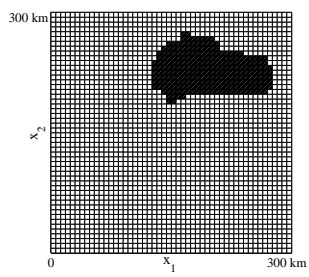

(c) $s(\chi)=460$

Fig. 1. Some samples of harvesting field configurations. The black areas correspond to protected regions, where $\chi=0$.

The harvested and protected regions are entirely determined by the harvesting field $\chi(\mathbf{x})$ present in (2) via the removal term $Y$. In order to build gradually fragmented configurations of these regions, we have discretized the domain $\Omega$ into $50 \times 50$ subcells $C_{i}$, with in some cells $\chi(\mathbf{x})=0$ for the unharvested cells, and $\chi(\mathbf{x})=1$ in the other cells. Based on the stochastic model of Roques and Stoica (2007), we have built 6000 samples of such functions $\chi(\mathbf{x})$, with different degrees of fragmentation. In all these samples, the protected region occupies $10 \%$ of the domain $\Omega$. The fragmentation of the protected region is defined as follows. The lattice made of the cells $C_{i}$ is equipped with a 4-neighborhood system $V\left(C_{i}\right)$. We set $s(\chi)=$ number of pairs of neighbors $\left(C_{i}, C_{j}\right)$ such that $\chi$ takes the value 0 on $C_{i}$ and $C_{j}$. This number $s(\chi)$ is directly linked to fragmentation: the protected region is all the more aggregated as $s(\chi)$ is high, and all the more fragmented as $s(\chi)$ is small (Fig. 1). Therefore, $s(\chi)$ can be seen as an "aggregation index" of the protected region. On our samples, the aggregation index $s$ varies from 94 to 460. For each aggregation index incremented as follows $s_{k}:=94+6 \times(k-1)$, we picked up arbitrarily a configuration $\chi_{k}$ with $s\left(\chi_{k}\right)=s_{k}$. This lead to 62 harvesting field distributions, with gradually aggregated configurations of the protected region.

\subsection{Methods}

The response to the spatial perturbation terms $Y(\mathbf{x}, u)$, distributed according to the aggregation index of their underlying harvesting fields, as described above, is analyzed in terms of total population size $P(t)$, and annual yield $R(t)$ in the region $\Omega$. More precisely, we evaluate the time-dependent quantities

$$
P(t):=\int_{\Omega} u(t, \mathbf{x}) \mathrm{d} \mathbf{x}
$$

corresponding to the total population at time $t$, and

$$
R(t):=\int_{t-1}^{t} \int_{\Omega} Y(\mathbf{x}, u(\tau, \mathbf{x})) \mathrm{d} \mathbf{x} \mathrm{d} \tau
$$

corresponding to the annual yield during the year that precedes $t$. 
Note 1: In the case of quasi-constant-yield harvesting, if $u(\tau, \mathbf{x})$ is greater or equal than $\varepsilon$ for all $\tau$ living within the temporal window $(t-1, t)$ and $\mathbf{x}$ in the domain $\Omega$, we simply obtain

$$
R(t)=\delta \cdot[\text { area of the harvested region] }
$$

In the case of proportional harvesting, we have:

$R(t)=E \cdot[$ mean population in the harvested region during the year $(t-1, t)]$.

We estimate the intertwined effects of fragmentation per se and harvesting intensity by plotting $P(t)$ and $R(t)$ against $\delta$ for the quasi-constant yield strategy, and against $E$ for the proportional harvesting strategy. For every configuration, the result is represented by a curve which color is attributed in function of the aggregation index; red corresponds to the more aggregated configurations, and blue to the more fragmented configurations. For each fixed harvesting intensity, we computed the gap between the maximum and minimum population sizes obtained over the 62 harvesting field distributions, and we expressed it in terms of relative loss obtained in the worst configuration compared to the best one, through the formula: $100 \times$ (highest population lowest population)/(highest population). Similarly, for the annual yield, $100 \times$ (highest annual yield - lowest annual yield)/(highest annual yield) was computed for each fixed harvesting intensity, where the maxima and minima are taken over the 62 harvesting field distributions.

For the numerical setup we consider $\Omega$ to be a square domain of $300 \mathrm{~km} \times 300 \mathrm{~km}$. We set $r=1$ year $^{-1}$ and $K=10^{3}$ individuals $/ \mathrm{km}^{2}$. The diffusion coefficient $D$ varies between $10 \mathrm{~km}^{2}$ /year (low mobility) and $100 \mathrm{~km}^{2} /$ year (high mobility); see the book of Shigesada and Kawasaki (1997) for some observed values of $r$ and $D$, for several animal species. The threshold $\varepsilon$ is set to 10 individuals $/ \mathrm{km}^{2}$. Our results, except in Section 3.4, are presented at a fixed time $t=5$; this time has been chosen to fit usual times for observations and responses in anthropic harvesting activities.

The numerical integrations of the RD models were performed using a second order finite elements method where the solutions $u(t, \mathbf{x})$ of the model (2) have been computed with the initial condition and harvesting strategies discussed above. The quantities of interest $P(t)$ and $Q(t)$ are then easily computable. The numerical results with ecological interpretations are discussed in the following section. 


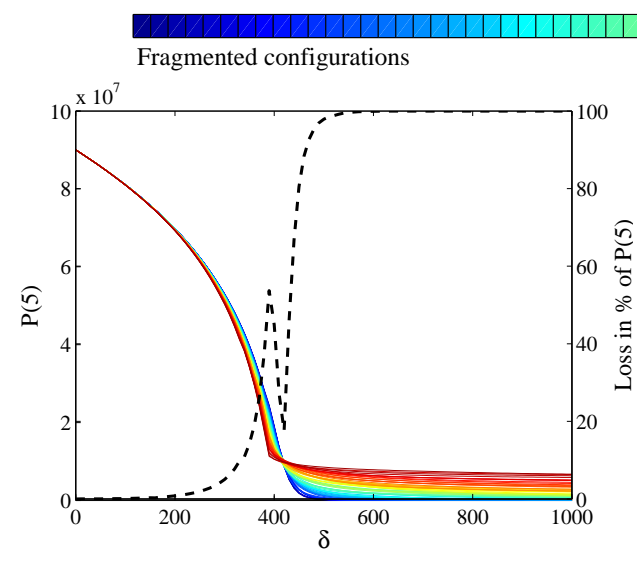

(a)

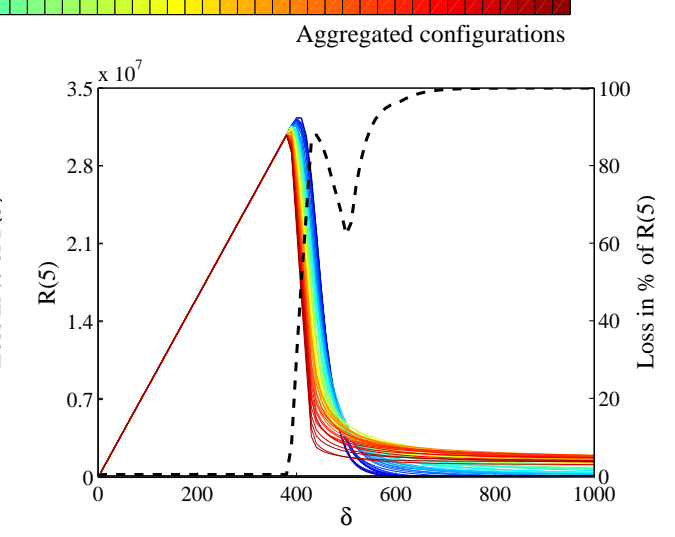

(b)

Fig. 2. (a): Total population in $\Omega$ after 5 years, in function of the quota $\delta$. (b): Total yield during year $4, R(5)$, in function of $\delta$. Each curve is associated with a different configuration of the protected region. Blue curves correspond to more fragmented configurations, and red curves correspond to more aggregated configurations. The black dotted lines indicate, for each $\delta$, the relative losses obtained in the worst configurations compared to the best ones. These computations were carried out for $D=50$.

\section{Results}

\subsection{Quasi-constant-yield harvesting strategy}

For every configuration, the higher the quota $\delta$, the smaller the population size $P(5)$ (Fig. 2, a). On the other hand, a maximum yield $R(5)$ is reached for an intermediate value of the quota, while small values and large values of $\delta$ both lead to small yields (Fig. 2, b).

As it could be expected, for small values of $\delta$, there is no dependence of the yield with respect to the habitat configuration. Indeed, in such cases, the population density should be everywhere above $\varepsilon$, and the harvesting function is therefore constant in time, equal to $\delta$ in the harvested regions; see Note 1. However, this linear dependence of $R(5)$ pursues for higher values of $\delta$ in fragmented configurations. This indicates that the population density never falls below $\varepsilon$ on these configurations. Thus, the more fragmented configurations first lead to higher yields for intermediate quotas, with a yield loss in aggregated configurations which attains $88 \%$. Conversely, the more fragmented configurations lead to lower yields for higher values of $\delta$. Overall, the maximum yield is attained on the more fragmented configuration. Similarly, population sizes are higher for fragmented configurations and low quotas, with losses in population size up to $54 \%$ on aggregated configurations. They then become higher for aggregated configurations and higher quotas. It is noteworthy that, 
whenever the harvesting term is really constant, i.e., in the region where $R(5)$ is linear, fragmented configurations lead to higher population sizes.

Such a reversion of the influence of fragmentation on both $P$ and $R$, for increasing quotas, is not intuitively obvious. Yet, we can give a reasonable explanation for it. In fragmented configurations, the mean distance to a protected region - where population density is higher — is reduced compared to more aggregated configurations. Therefore, when $\delta$ is not too large, at each location in $\Omega$, the population can be efficiently sustained by the protected regions, and the density never falls below the threshold $\varepsilon$, leading to higher yields, compared to more aggregated configurations. For larger values of $\delta$, the harvested regions become very hostile, and because of dispersion, populations tend to extinct, even in the protected regions. In the case of protected regions with small perimeters, corresponding to aggregated configurations, dispersion of the individuals into the harvested regions is reduced. With such configurations, populations can therefore sustain higher quotas without risking extinction, leading to higher values of $P$ and $R$. Note that, whenever $u(t, \mathbf{x})$ is less than or equal to $\varepsilon$ everywhere in the harvested region, quasi-constant-yield harvesting becomes equivalent to proportional harvesting, with effort $E=\delta / \varepsilon$.

Comparable qualitative results were found for diffusion coefficients $D$ ranging from 10 to 100 and are thus not shown.

\subsection{Proportional harvesting strategy}

When the harvesting function $Y$ is of proportional type (4), our model reduces to $\frac{\partial u}{\partial t}-D \nabla^{2} u=r u(1-E \cdot \chi(\mathbf{x}) / r-u / K)$. Population persistence for this model has been thoroughly investigated (Cantrell and Cosner, 2003; Berestycki et al., 2005a; Roques and Hamel, 2007; Roques and Stoica, 2007), both analytically and numerically.

The specific effects of fragmentation of the protected regions can be deduced from the numerical study of Roques and Stoica (2007). It shows that, on aggregated configurations, higher efforts $E$ can be sustained without risking extinction. The optimal shapes of the protected regions, in terms of maximum sustainable effort, has even been obtained in (Roques and Hamel, 2007). Surprisingly, these shape depend on the area of the protected region. Indeed, small areas have been proved to lead to disc-shaped optimal shapes, while high areas lead to stripe-shaped optimal shapes.

Following our approach, we still focus on the quantities $P$ and $R$, on which the effects of fragmentation have not yet been investigated. For every configuration, the population size $P(5)$ decreases as the effort $E$ increases (Fig. 3,

a). Contrarily to the quasi-constant case, with such a proportional harvesting 


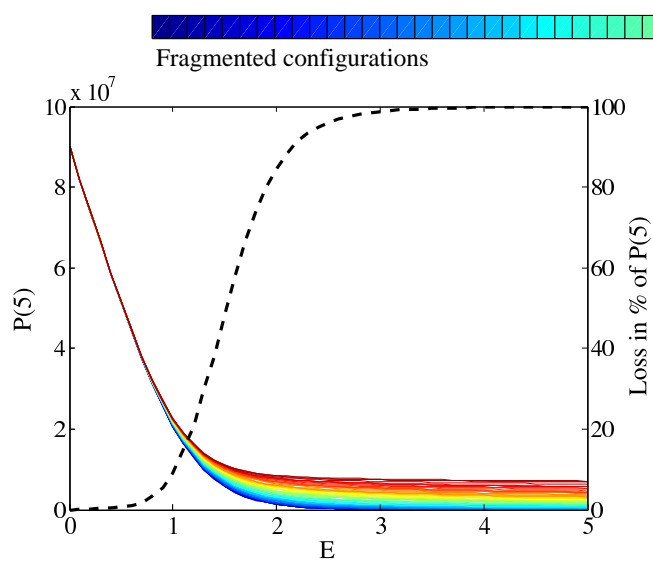

(a)

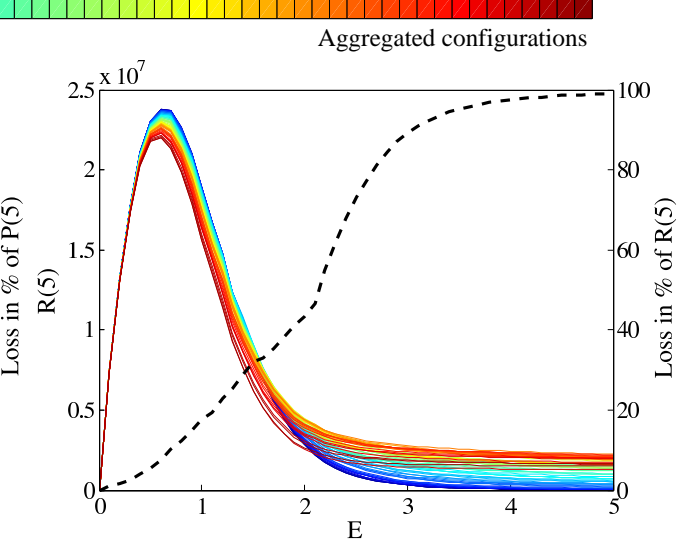

(b)

Fig. 3. (a): Total population in $\Omega$ after 5 years, in function of the harvesting effort $E$. (b): Total yield during year $4, R(5)$, in function of $E$. To each curve corresponds a different configuration of the protected regions; the blue curves correspond to more fragmented configurations, and the red curves correspond to more aggregated configurations. The black dotted lines indicate, for each value of $E$, the relative losses obtained in the worst configurations compared to the best ones. For these computations, we fixed $D=50$.

strategy, aggregated configurations always lead to larger populations, whatever the effort. The effect of fragmentation/aggregation of the protected region on the population sizes really becomes noticeable when $P(5)$ falls below one fourth of the environment carrying capacity. In such a case, the involved mechanisms are those of persistence, which require in particular the state 0 , where no individuals are present, to be very repulsing (or equivalently "unstable", see Shigesada and Kawasaki, 1997; Berestycki et al., 2005a). It is therefore not surprising to obtain effects of fragmentation comparable to those described in the existing literature.

On the other hand, the yield $R(5)$ again reaches a maximum (cf. Fig. 3, b). As in the quasi-constant-yield case, the maximum yield is attained for the most fragmented configuration. Fragmented configurations have a longer perimeter, and thus provide higher transfer rates into the harvested regions. Indeed, obtaining higher yields with smaller total populations, for small values of $E$, implies that the size of the population situated outside the protected regions is higher for fragmented configurations; see Note 1. In the quasi-constant case, such a larger "unprotected" population would not have implied higher catches whenever $u$ greater than $\varepsilon$; this explains the qualitative difference between the two harvesting strategies in terms of the effects of fragmentation on $P(5)$.

As above discussed, higher values of the effort give a significant advantage to aggregated configurations, in terms of total population sizes. This translates into higher yields on aggregated configurations. Still, these qualitative results do not depend on the values of diffusion coefficients, in the selected range 


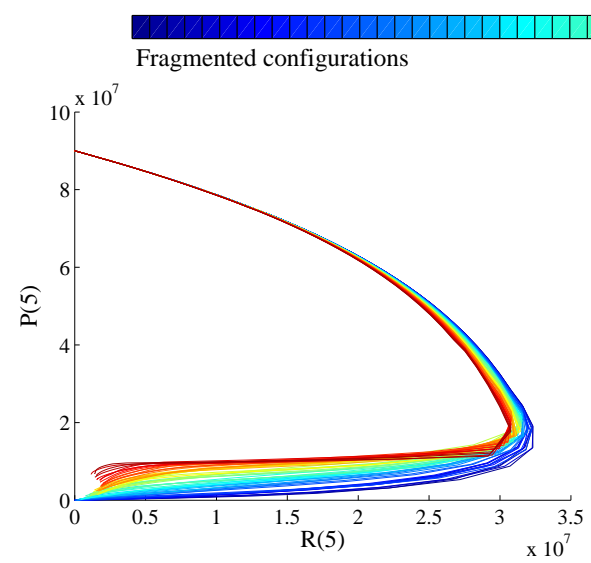

(a)

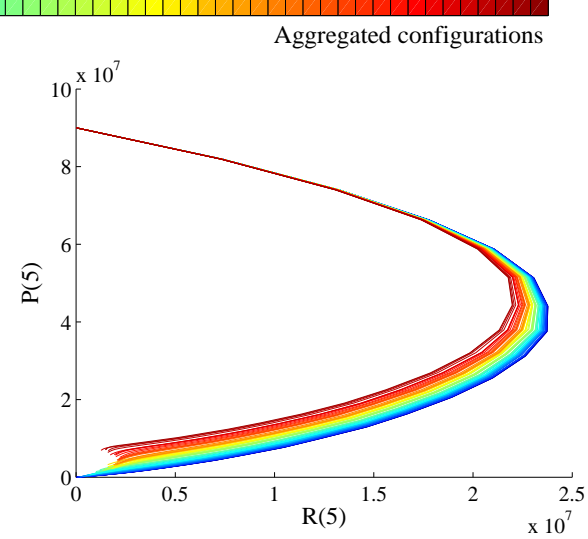

(b)

Fig. 4. (a): Total population $P(5)$ in $\Omega$ after 5 years, in terms of the yield during year $4, R(5)$, for the quasi-constant-yield strategy. (b): Total population $P(5)$ vs yield $R(5)$, for the proportional harvesting strategy. Blue curves correspond to more fragmented configurations, and red curves correspond to more aggregated configurations. For these computations, we fixed $D=50$.

$[10,100]$, and are not shown.

\subsection{Total population vs. annual yield}

In Fig. 4, are depicted the population sizes $P(5)$, in terms of the yields $R(5)$, and of the level of fragmentation of the protected region for the quasi-constantyield and proportional harvesting strategies (Figs. 4, (a) and (b), respectively). The flip shapes of these diagrams teach us that for a given yield two branch of disjoint intervals of population size are admissible, sufficiently far to the left from the "bending point". The upper branch corresponds to low harvesting intensities (below that leading to maximum yield), and the lower branch corresponds to higher intensities. Remarkably, for each given population size, higher yields are obtained on more fragmented configurations; the lower the population size, the higher this effect.

This $\supset$-shape of the $P-R$ diagram supports the idea that it will be difficult to predict the quantitative effect of fragmentation in practice, at a fixed yield, without knowledge of the total population size; a situation which typically arises in ecological application where such a knowledge is difficult to achieve.

\subsection{Results for times $t \in(0,20)$}

Fig. 5 depicts how the total population $P(t)$ depends on the harvesting intensity and on the aggregation index in function of the time $t$. 


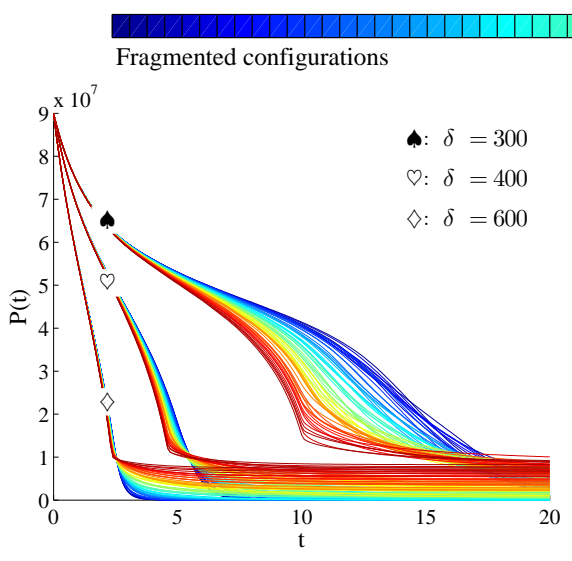

(a)

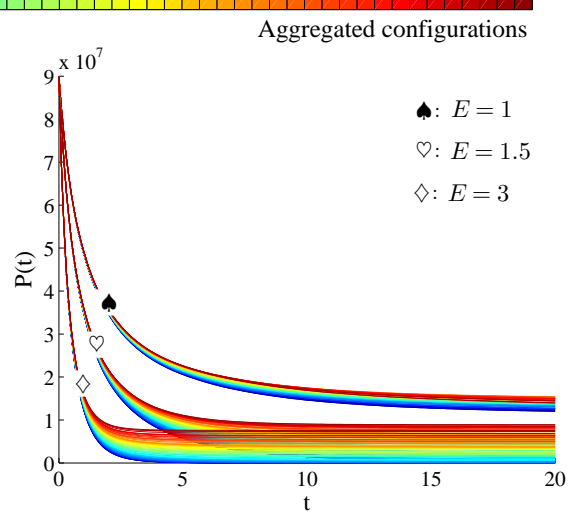

(b)

Fig. 5. (a): Total population $P(t)$ in function of time $t$ and in terms of the quota $\delta$, for the quasi-constant-yield strategy. (b): Total population $P(t)$ in function of time $t$ and in terms of the effort $E$, for the proportional harvesting strategy. For these computations, we again fixed $D=50$.

For the quasi-constant-yield harvesting strategy (Fig. 5, a), we observe that the higher the quota, the sooner the inversion of the effects of fragmentation. Thus, the threshold quotas, above which aggregated configurations lead to higher populations, decrease as $t$ increases.

For the proportional harvesting strategy (Fig. 5, b), at each time $t$, aggregated configurations are still associated with larger populations. Moreover, the effect of fragmentation tends to increase with time.

In both cases, the amplitude of the effect of fragmentation depends on the harvesting intensity and on the time point of the analysis. However, those results, and in particular the quasi-constant-yield case, suggest previous sections results might still be qualitatively true at times other that $t=5$. This is confirmed by numerical computations (not presented here) which indeed lead to comparable results, but with a shift in the harvesting intensity.

Analytical studies show that the solution $u(t, x)$ of (2) converges to some equilibrium state for both quasi-constant-yield (Roques and Chekroun, 2007) and proportional (Berestycki et al., 2005a) harvesting strategies; however, convergence rates are not known. Figs. 5 (a) and (b) provide information about these convergence rates: the higher the harvesting intensities, the sooner equilibria are reached. Thus, at $t=5$, population sizes may be almost at equilibrium for high harvesting intensities or still farer to reach it for lower intensities. 


\section{Concluding remarks}

In the existing single-species modeling literature, fragmentation of the favorable region is usually found to be detrimental for population survival. On the other hand, recent empirical studies show that fragmentation may have positive or negative effects, and that positive effects occur more often.

In her review paper on the topic Fahrig (2003) proposed two reasons for negative effects of fragmentation of these favorable regions. Firstly, favorable patches are too small to sustain a local population, and secondly, the total perimeter of the favorable region is large, leading to increased transfer rates into the unfavorable regions. She also proposed several reasons for positive effects of fragmentation. Among these reasons, smaller distance between patches, higher immigration rates into the patches (see Grez et al., 2004) and positive edge effects for some species are evoked.

In our work, based on single-species reaction-diffusion models with harvesting terms, we have captured both positive and negative effects of fragmentation of the favorable region, interpreted here as a protected region.

Firstly, for large harvesting terms, we found that aggregated configurations of the protected region lead to higher population sizes, and higher yields than fragmented configurations. In that sense, our results are not contradictory with previous modeling results: under hostile conditions, the extinction risks are higher, and the chances of persistence are increased on aggregated configurations. The reasons evoked by Fahrig for negative effects of fragmentation then become paramount in our model at low population sizes.

On the other hand, fragmented protected regions lead to higher population sizes when a constant number of individuals are removed per unit of time in the unprotected region (constant-yield-harvesting). Furthermore, for fixed population sizes, the two harvesting strategies we studied in this paper lead to higher yields for fragmented configurations; cf. Fig. 4. Such higher yields may stimulate harvesters to slow down, and may therefore be beneficial for populations.

Sumaila (1998), through a compartment modeling approach, showed that transfer rates between favorable and unfavorable regions were of critical importance for understanding the role of reserves, and the author emphasized the necessity of a more precise modeling approach of the transfer rate function. Latter, Tischendorf et al. (2005) found, via a simulation approach, negative effects of fragmentation when the probability of individuals to go from the favorable to the unfavorable region was high, and positive effects in the opposite case with a high unfavorable to favorable boundary-crossing probability. In our models, the diffusion coefficient is spatially-constant, and the boundary- 
crossing probabilities are therefore equal in inward and outward directions. However, net transfer rates from favorable to unfavorable regions depend on the geometry of the protected regions, and on the relative population densities inside and outside the favorable regions. Indeed, individuals moves are driven by random diffusion, and Green's formula (see e.g. Evans, 1998) implies that the instantaneous population flux from the protected region to the harvested one is:

$$
\text { Flux Protected region } \rightarrow \text { harvested region }=-D \int_{\Gamma} \frac{\partial u}{\partial n} d s,
$$

where $\Gamma$ denotes the boundary of the protected region, and $\frac{\partial u}{\partial n}$ is the outward gradient in population density observed on this boundary. Our work shows that both the geometry of the protected regions, and the relative population densities inside and outside the favorable regions interact to give negative effects of fragmentation for high harvesting terms, i.e. when the contrast between protected and harvested areas is strong, and positive effects for less contrasted environments.

In multi-species models, fragmentation per se can alter interactions among species. As reviewed by Ryall and Fahrig (2006), predator-prey models predict varying effects of fragmentation on equilibrium densities of predator and prey populations, depending on the specific assumptions of these models. In this paper we have demonstrated that single-species reaction-diffusion models with removal terms can support relative effects of fragmentation per se. The methods and results could serve as a first step to bridge the gap between empirical work and modeling, for reaction-diffusion as well as other single-species models.

\section{Acknowledgements}

The authors are indebted to an anonymous referee and to André Kretzschmar

for their valuable comments. The authors are supported by the French "Agence Nationale de la Recherche" within the projects PREFERED, ColonSGS (first author) and URTICLIM (first author).

\section{References}

Beddington, J. R., and R. M. May. 1977. Harvesting natural populations in a randomly fluctuating environment. Science 197:463-465.

Berestycki, H., F. Hamel, and L. Roques. 2005a. Analysis of the periodically fragmented environment model: I-Species persistence. Journal of Mathematical Biology $51: 75-113$. 
Berestycki, H., F. Hamel, and L. Roques. 2005b. Analysis of the periodically fragmented environment model: II-Biological invasions and pulsating travelling fronts. Journal de Mathématiques Pures et Appliquées 84:1101-1146.

Bolker, B. M. 2003. Combining endogenous and exogenous spatial variability in analytical population models. Theoretical Population Biology 64:255-270.

Cantrell, R. S., and C. Cosner. 1989. Diffusive logistic equations with indefinite weights: population models in disrupted environments. Proceedings of the Royal Society of Edinburgh 112:293-318.

Cantrell R. S., and C. Cosner. 2003. Spatial Ecology via Reaction-Diffusion Equations. Ser. Math. Comput. Biol., John Wiley and Sons, Chichester, UK.

Chekroun M. D., and L. J. Roques. 2006. Models of population dynamics under the influence of external perturbations: mathematical results. Comptes Rendus Mathematique, Acadadémie des Sciences de Paris, Serie I 343:307-310.

Christensen C., S. Guénette, J. J. Heymans, C. J. Walters, R. Watson, D. Zeller, and D. Pauly. 2003. Hundred-year decline of North Atlantic predatory fishes. Fish and Fisheries 4:1-24.

El Smaili, M., F. Hamel, and L. Roques. 2009. Homogenization and influence of fragmentation in a biological invasion model. Discrete and Continuous Dynamical Systems Series A, 25:321-342.

Evans, L. C. 1998. Partial Differential Equations. University of California, Berkeley - AMS.

Fahrig, L. 2003. Effects of habitat fragmentation on biodiversity. Annual Review of Ecology, Evolution and Systematics, 34:487-515.

Fisher, R. A. 1937. The advance of advantageous genes. Annals of Eugenics, 7:335369.

Gardner, R. H., B. T. Milne, M. G. Turner, and R. V. O’Neill. 1987. Neutral models for the analysis of broad-scale landscape pattern. Landscape Ecololgy 1:19-28.

Grez, A., T. Zaviezo, L. Tischendorf, and L. Fahrig. 2004. A transient, positive effect of habitat fragmentation on insect population densities. Oecologia 141:444-451

Hale, J. K., and S. M. Verduyn Lunel. 1990. Averaging in infinite dimensions. Journal of Integral Equations and Applications 2:463-494.

Holmes, E.E. 1993. Are Diffusion Models too Simple? A Comparison with Telegraph Models of Invasion. The American Naturalist 142:779-795.

Huffaker, C.B. 1958. Experimental Studies on Predation: Dispersion factors and predator-prey oscillations. Hilgardia 27:343-383.

Keitt, T. H. 2000. Spectral representation of neutral landscapes. Landscape Ecology 15:479-494.

Kinezaki, N., K. Kawasaki, F. Takasu, and N. Shigesada. 2003. Modeling biological invasion into periodically fragmented environments. Theoretical Population Biology 64:291-302.

Kolmogorov, A. N., I. G. Petrovsky, and N. S. Piskunov. 1937. Etude de l'équation de la diffusion avec croissance de la quantité de matière et son application à un problème biologique, Bulletin Université d'État à Moscou (Bjul. Moskowskogo Gos. Univ.), Série internationale A 1:1-26.

Mandelbrot, B. B. 1982. The Fractal Geometry of Nature, W. H. Freeman, New York.

Murray, J. D. 2002. Mathematical biology. Third edition, Interdisciplinary Applied 
Mathematics 17, Springer-Verlag, New York.

Neubert, M. G. 2003. Marine reserves and optimal harvesting, Ecology Letters 6:843-849.

Okubo, A., and S. A. Levin. 2002. Diffusion and Ecological Problems-Modern Perspectives. 2nd ed., Springer-Verlag, New York.

Oruganti, S., R. Shivaji, and J. Shi. 2002. Diffusive logistic equation with constant effort harvesting, I: Steady states, Transactions of the American Mathematical Society 354:3601-3619.

Pearson, K., and J. Blakeman. 1906. Mathematical Contributions of the Theory of Evolution, XV, A Mathematical Theory of Random Migration. Drapersi Company Research Mem. Biometrics Series III, Dept. Appl. Meth. Univ. College, London.

Robinson J. G., and R. E. Bodmer. 1999. Towards wildlife management in tropical forests. Journal of Wildlife Management 63:1-13.

Robinson J. G. and K. H. Redford. 1991. Sustainable harvest of neo-tropical mammals. Pages 415-429 in J. G. Robinson and K. H. Redford, eds. Neo-Tropical Wildlife Use and Conservation, Chicago University Press, Chicago, IL.

Roques, L., and M. D. Chekroun. 2007. On population resilience to external perturbations. SIAM Journal on Applied Mathematics 68:133-153.

Roques, L., and F. Hamel. 2007. Mathematical analysis of the optimal habitat configurations for species persistence. Mathematical Biosciences 210:34-59.

Roques, L., and R. Stoica. 2007. Species persistence decreases with habitat fragmentation: An analysis in periodic stochastic environments. Journal of Mathematical Biology 55:189-205.

Ryall, K. L., and L. Fahrig. 2006. Response of predators to loss and fragmentation of prey habitat: a review of theory. Ecology 87:1086-1093.

Saunders, D.A., R. J. Hobbs, and C. R. Margules. 1991. Biological consequences of ecosystem fragmentation: a review. Conservation Biology 51:18-32.

N. Shigesada and K. Kawasaki,. 1997. Biological Invasions: Theory and Practice. Oxford Series in Ecology and Evolution, Oxford University Press, Oxford, UK.

Skellam, J. G. 1951. Random dispersal in theoretical populations. Biometrika 38:196-218.

Stephens, P. A., F. Frey-Roos, W. Arnold, and W. J. Sutherland. 2002. Sustainable exploitation of social species: A test and comparison of models. Journal of Applied Ecology 39:629-642.

Sumaila, U. R. 1998. Protected marine reserves as fisheries management tools: a bioeconomic analysis, Fisheries Research 37:287-296.

Tischendorf, L., A. Grez, T. Zaviezo, and L. Fahrig. 2005. Mechanisms affecting population density in fragmented habitat. Ecology and Society 10:7.

Turchin, P. 1998. Quantitative Analysis of Movement: Measuring and Modeling Population Redistribution in Animals and Plants, Sinauer Associates, Sunderland, MA. 